\title{
La medición del desempeño social empresarial a través de las redes sociales
}

Julio Sesma

Tecnológico de Monterrey

julio.sesma@gmail.com

Bryan Husted

York University, Schulich School of Business y Tecnológico de Monterrey bryanhusted@gmail.com

\section{Jerry Banks}

Tecnológico de Monterrey atljerry@yahoo

\section{Resumen}

El desempeño social empresarial (CSP, por sus siglas en inglés) ha sido un tema ampliamente estudiado en el campo de la administración. Sin embargo, su método de medición continúa siendo ambiguo, genera controversia y, al mismo tiempo, es complejo para la investigación. Este trabajo propone la medición del CSP, definido como la satisfacción de los stakeholders, a través de las redes sociales. Consideramos que la satisfacción de los stakeholders puede ser entendida utilizando elementos de la teoría de justicia organizacional, particularmente en lo referente al uso de la expresión de voz de los stakeholders ante un evento de injusticia por parte de las empresas. El crecimiento de las redes sociales, y específicamente de los microblogs, ha permitido que las personas puedan expresar su opinión respecto a los eventos económicos, sociales, políticos y ambientales de cualquier tipo de empresa. Probamos nuestras ideas utilizando la metodología de estudio de eventos con una muestra de 5440 datos provenientes de diez empresas; se encontró evidencia de la sensibilidad a estos eventos en las redes sociales.

Palabras clave: desempeño social empresarial, stakeholder, redes sociales, análisis de los sentimientos, estudio de eventos. 


\title{
Measuring corporate social performance through social media
}

\begin{abstract}
Corporate social performance (CSP) has been studied extensively by management scholars, yet most approaches to its measurement continue to be ambiguous, controversial and difficult to research. In this paper, we propose measuring CSP via the construct of stakeholder satisfaction through social media. Using organizational justice theory, we argue that the satisfaction of stakeholder expectations can be understood partly through the perception of unjust corporate behavior, which is then expressed by stakeholders through social media. The explosion of social networking and microblogging has made it possible for people to express opinions with respect to the economic, social, and environmental events for all sorts of firms. We test these ideas using event study methods with a sample of 5,440 observations from ten firms. We find preliminary evidence of the sensitivity of social media to these events.
\end{abstract}

Keywords: corporate social performance; stakeholder; social media; sentiment analysis; event study

\section{Introducción}

La literatura sobre el desempeño social de las empresas (CSP, por sus siglas en inglés) ha sido un tópico relevante en la administración estratégica de las mismas, dado el continuo supuesto de la existencia de una relación con el desempeño financiero de las empresas (CFP) (Waddock y Graves, 1997; Berman et al., 1999; Harrison y Freeman, 1999; Luoma y Goodstein, 1999; Harrison, Bosse y Phillips, 2010; Alonso-Almeida et al., 2012); sin embargo, dicho supuesto continúa siendo ambiguo y sin consenso (Briseño García, Lavín Verástegui y García Fernández, 2011). Asimismo, conocer si existe una relación positiva o negativa entre el CSP y el CFP no ayuda a comprender cómo las empresas y sus stakeholders reaccionan ante una situación o evento relacionado a su CSP (Rowley y Berman, 2000).

Dicha inconsistencia representa precisamente el principal motivo para este trabajo. Se propone el estudio del CSP a través del análisis de los stakeholders (Preston, 1990; Wood y Jones, 1995; Clarkson, 1995; Harrison y Freeman, 1999; Husted, 2000; Rowley y Berman, 2000; Bosse et al., 2009; Wartick y Mahon, 2009; Orlitzky y Swanson, 2012). Se define como stakeholders a aquellos que influyen en la empresa o son afectados por ella (Freeman, 1984); es decir, empleados, accionistas, clientes, proveedores, competidores, gobiernos, comunidades y organiza- 
ciones no-gubernamentales. Se entiende por CSP el impacto que la empresa tiene sobre sus stakeholders (Clarkson, 1995; Wood y Jones, 1995); para Clarkson (1995) el CSP tiene que ser evaluado con base en la interacción de la empresa con los diferentes stakeholders. Lo más importante es que los resultados puedan ser claramente evaluados y no simplemente descritos (Clarkson, 1988).

Igualmente, la literatura referente al CSP y su relación con los stakeholders ha llegado a la conclusión de que la satisfacción de las expectativas de éstos puede ser un indicador clave en la medición del CSP (Mitnick, 2000). En el presente estudio se explica la satisfacción de las expectativas de los stakeholders ante eventos específicos a través de la teoría de justicia organizacional. Dado lo anterior, si las expectativas de los stakeholders no son las esperadas, éstos pueden manifestar su insatisfacción ante el desempeño de las empresas. De manera particular se considera la opinión o voz de los stakeholders ante determinada acción de la empresa, que puede ser percibida como injusta. El crecimiento de las redes sociales y microblogs ha hecho posible que las personas puedan expresar su opinión con respecto al comportamiento de las empresas con mayor facilidad.

De igual manera, el inmenso volumen de información existente en las redes sociales puede contribuir significativamente en la identificación de elementos que permitan medir la reacción de los stakeholders ante las actividades de una empresa en específico. Se evalúa la satisfacción o insatisfacción a través del sentimiento del texto que emite el stakeholder en una red social. Este sentimiento se puede definir como una actitud positiva o negativa, la cual es desarrollada por una persona o un grupo de personas hacia una entidad en específico, la cual puede ser una persona, un grupo, una organización, una ubicación geográfica, un evento, una acción que implica a las entidades, así como una idea o concepto (Fink, Chou, Kopecky y Llorens, 2011).

Para determinar la valencia global de los sentimientos emitidos en un texto o en un día, se utiliza el concepto de la polaridad del mismo. Por otra parte, se utilizan los elementos metodológicos del estudio de eventos y de la regresión polinomial local para identificar y examinar los datos "anormales" en la polaridad de los sentimientos de los stakeholders ante eventos específicos realizados por parte de las empresas, los cuales son expresados en diversas redes sociales como Twitter.

El artículo está organizado, además de esta introducción, en tres secciones. En primer lugar se explican los elementos conceptuales que sustentan la investigación; 
de manera particular se expone la lógica conceptual en donde se vincula la observación de un acto injusto por parte de los stakeholders y su expresión de satisfacción o insatisfacción en las redes sociales. Después se presentan los métodos para analizar las redes sociales como una expresión de satisfacción o insatisfacción. Finalmente se presentan los principales resultados de la investigación.

\section{Marco teórico}

La literatura de la justicia organizacional se enfoca principalmente en comprender cómo los empleados evalúan las decisiones y procesos de toma de decisiones ante acciones específicas de la empresa (Leventhal, 1976; Lind y Tyler, 1988). Para establecer el grado de justicia de determinada decisión se requiere la evaluación de dos principios específicos (Sheppard et al., 1992). El primero hace énfasis al proceso de "balance", el cual se refiere a la comparación entre una acción determinada que se compara a otra similar; dicha acción genera "resultados" que son evaluados y finalmente percibidos como "justos o injustos" (Adams, 1965). El segundo, es el principio de lo "correcto", el cual se refiere a la calidad en el proceso que hace a una decisión parecer como "correcta", también conocido como la "justicia procedimental". Desde la perspectiva de los stakeholders este último principio hace énfasis al proceso realizado entre la persona que toma una decisión y la persona afectada por el resultado de dicha decisión.

Los procesos son tan importantes para la teoría de los stakeholders como la distribución final de costos y beneficios de la decisión. La percepción de la incongruencia, ambigüedad, inmoralidad o la falta de procesos claros, puede reflejar una carencia de procesos correctos (Sheppard et al., 1992). Sin embargo, en algunas ocasiones los stakeholders tienen la necesidad de responder emocionalmente para tratar de reducir su estrés o percepción de daños causados por un acto de injusticia; por lo tanto, se considera que siguen patrones de respuesta dinámica o emocional (Sheppard et al., 1992) que puede ser resultado de una sencilla evaluación de costo-beneficio cuando se considera que el daño no es tan relevante o cuando la diferencia entre el costo y el beneficio no es tan significante.

\section{Justicia organizacional y la teoría de los stakeholders}

Investigaciones recientes en el área de la administración se han enfocado a entender la relación entre la administración de los stakeholders y la teoría de la justicia organizacional. Harrison, Bosse y Phillips (2010) destacan la importancia de la 
justicia organizacional en el proceso de evaluación de los stakeholders que se encuentran fuera de la organización, los cuales también pueden ser clasificados como terceros, concepto que hace referencia principalmente a aquellos individuos que se forman una impresión ante un evento realizado por parte de una empresa especifica hacia otra persona o grupo de personas (Skarlicki y Kulik, 2005). Los terceros también se pueden definir como aquellos individuos que no se encuentran directamente afectados por el acto de injusticia. Dentro de este grupo se pueden clasificar al público en general, inversionistas, clientes, compañeros de oficina, empleados de gobierno o cualquier otro actor que observa un acto de injusticia hacia una persona o grupo de personas (O'Reilly y Aquino, 2011).

Cabe señalara que los terceros representan una importante fuente de información acerca de los actos de injusticia cometidos por parte de las empresas hacia sus stakeholders. O'Reilly y Aquino (2011) mencionan que aquéllos pueden reaccionar ante los eventos de injusticia de diversas maneras; por ejemplo, algunos elaboran anuncios públicos que puedan generar cualquier tipo de acción que afecte a los perpetradores del acto de injusticia o buscan castigar los actos de injusticia por su propia cuenta; otros representan una fuente de apoyo a las víctimas que sufren de malos tratos al proporcionar algún tipo de apoyo emocional dando consejos o simplemente escuchándolos; unos más simplemente pueden decidir no hacer nada.

La literatura actual sobre terceros ha identificado dos principales corrientes de investigación respecto a sus motivaciones: una explica el actuar de los terceros con base en su propio interés; la otra concibe el actuar de los terceros como un acto de interés moral (Skarlicki y Kulik, 2005; O’Reilly y Aquino, 2011). El interés personal se basa en los supuestos de que las personas son motivadas por un interés "racional" o económico en su relación con los otros miembros de la sociedad; un ejemplo de lo anterior es la teoría del intercambio social (Blau, 1964) que propone que las personas siguen ciertas normas de cambio dado que cuentan con un interés personal. Por su parte, la teoría de la equidad (Adams, 1965) menciona que las personas pueden experimentar sufrimiento o preocupación cuando la percepción o balance entre lo que se ofrece, comparado con lo que se recibe, es menor a lo esperado. Asimismo, el modelo instrumental de la justicia procedimental propone que las personas se preocupan sobre los procesos justos con el propósito de maximizar sus utilidades (Thibaut y Walker, 1975).

Investigaciones recientes enfocadas a la justicia organizacional han encontrado que la percepción de justicia va más allá de sólo el propio interés económico, pues 
sugieren que aquélla es un estándar universal de conducta interpersonal en donde algunas veces los stakeholders tienen una motivación moral involuntaria hacia los actos de injusticia, aun cuando ellos mismos no se encuentren directamente afectados. Dicho proceso es conocido como justicia deóntica (Rupp et al., 2006) que se refiere al estado psicológico que produce un cambio emocional ante aquellos eventos que violen las reglas percibidas del comportamiento social (Folger, 2001). La principal contribución del modelo de justicia deóntica es que explícitamente identifica la reacción de los "otros" actores no afectados como resultado de un comportamiento moral (O'Reilly y Aquino, 2011). Los terceros pueden estar dispuestos a sacrificar ganancias económicas con el propósito de castigar a aquellos que han cometido un acto de "injusticia", aun sin conocer a la víctima o recibir alguna compensación a cambio (Turillo et al., 2002).

\section{La reacción de terceros y el análisis de los sentimientos en las redes sociales}

Con el propósito de identificar la percepción de injusticia o insatisfacción por parte de los stakeholders como respuesta a un evento especifico ambiental o social que genera un impacto negativo sobre otros, en este trabajo se considera en el estudio del análisis de los sentimientos, que se refiere al estudio de técnicas computacionales de extracción y clasificación de textos, así como de su polaridad (ya sea positiva o negativa). Sin embargo, algunos autores amplían dicho concepto al incluir el tratamiento computacional de las opiniones, sentimientos y subjetividad en los textos (Pang y Lee, 2008).

El concepto de redes sociales se refiere al grupo de aplicaciones basadas en la Internet y que son creadas con las bases tecnológicas e ideológicas de la Web 2.0. Dichas bases permiten la creación y el intercambio de los contenidos generados por los usuarios o UGC (user-generated content), los cuales de acuerdo con la Organización para la Cooperación y el Desarrollo Económico (OECD, 2007) requieren alcanzar tres elementos básicos para ser considerado como tales: primero, dicho contenido tiene que ser publicado en un sitio de acceso público o dentro de una red social con acceso a un grupo selecto de personas; segundo, requiere mostrar cierto grado de creatividad; y tercero, necesita ser elaborado en un ambiente fuera del entorno profesional (Kaplan y Haenlein, 2010).

Posiblemente uno de los principales retos que tienen los investigadores de esta área es identificar todos aquellos elementos que puedan ser considerados como "señales" claras de información y no sólo "ruido". Una señal es definida como cualquier 
información que es relevante o de interés para los usuarios; por su parte, "ruido" es cualquier otra información que no es del interés de los usuarios.

En el contexto del análisis de los sentimientos, se entiende como señales a todo aquello que cambia la polaridad de los stakeholders. La polaridad es una medida del agregado de sentimientos, ya sean positivos, negativos e incluso neutrales, dentro de un texto determinado (Pang y Lee, 2008). Aun cuando este concepto parece sencillo, en la práctica los investigadores enfrentan un sinnúmero de retos al momento de realizar la evaluación computacional de la polaridad (Kale, et al. 2007).

Dentro de los textos que pueden ser sujetos de evaluación dentro del campo del análisis de los sentimientos se encuentran los microblogs, que son comentarios cortos comúnmente realizados dentro de un grupo social o una red social. La aplicación más popular de microblogs es Twitter (Jensen et al., 2009; Merlo et al., 2010); su popularidad en el campo del análisis de los sentimientos se da principalmente debido a la simplicidad de su interfaz de aplicación y programación (API), la cual permite a los investigadores tener acceso a millones de tweets (mensaje de texto que se emite por medio de Twitter) para su análisis de manera cronológica y geográfica.

Desde su lanzamiento el 13 de julio del 2006, Twitter se ha convertido en uno de los medios sociales de mayor crecimiento en el nivel mundial; se estima que contaba con más de 500 millones de usuarios en febrero de 2012 (Dugan, 2012). De acuerdo con Dick Costolo, CEO de Twitter, para junio de 2012 este microblog ha llegado a la cifra de 400 millones de tweets por día, lo que representa un incremento del 18\% en relación con mayo del mismo año (Bennett, 2012). Estos datos nos muestran el impresionante ritmo de crecimiento que tiene esta plataforma en relación con otras similares.

Cada día más empresas utilizan a Twitter como un nuevo canal para promover sus productos y servicios, así como para recomendar "seguir" a compañías especificas que pueden ser del interés de los usuarios dependiendo de sus perfiles (Thongsuk, Haruechaiyasak y Somkid, 2011). Sin embargo, hay un creciente interés en el estudio de las relaciones entre los eventos y los cambios en los sentimientos de los tweets. Algunos de estos estudios se enfocan en la relación que hay entre los precios de las acciones de las empresas y los sentimientos expresados en Twitter sobre la misma entidad (Thelwall, Buckley y Paltoglou, 2011). 
Por otra parte, la literatura actual sobre la investigación del análisis de los sentimientos examina la correlación entre eventos sociales, políticos, culturales y económicos y su relación con los sentimiento de los tweets (Thelwall y Prabowo, 2007). O'Connor, Balasubramanyan, Routledge, y Smith (2010) encontraron una fuerte correlación entre los resultados de preguntas colocadas en sitios de opinión y el sentimiento de los tweets durante el periodo de 2008 a 2009; estos autores recomendaron que la detección automática de los sentimientos puede servir como un monitor de la opinión pública (de manera positiva o negativa) en aquellos temas de importancia, tanto para las empresas como para aquellos temas populares (O’Connor et al., 2010).

Hasta el momento no se ha realizado algún trabajo que utilice Twitter como herramienta en la medición del desempeño social de las empresas o para el estudio de las reacciones de los stakeholders ante actos de injusticia. Lo anterior es relevante dado que existen múltiples publicaciones enfocadas a estudiar el análisis de los sentimientos a través de Twitter y su relación con diversos eventos sociales. Sin embargo, ninguna de ellas se enfoca a explicar cómo dichos sentimientos son resultado de determinados actos de injusticia percibida por los stakeholders directa e indirectamente afectados y de cómo dicho cambio puede ser expresado a través del uso de Twitter.

Las percepciones de injusticia son típicamente manifestadas en cambios en la actitud de los stakeholders hacia los autores de la injusticia. Asimismo, la injusticia o inequidad genera pequeños, pero importantes cambios en las actitudes de los stakeholders (Adams, 1965). Dada la amplia disponibilidad de las redes sociales, estos cambios de actitud se reflejarán en los textos emitidos por los stakeholders usuarios de Twitter.

Hipótesis: Los eventos de impacto social realizados por parte de las empresas y reportados en los medios de información producen cambios significativos en la polaridad de los sentimientos de los stakeholders, los cuales son expresados a través de Twitter. 


\section{Método}

\section{Medición de variables}

Proponemos utilizar Twitter como una nueva forma de medir las reacciones de los stakeholders ante eventos positivos y negativos realizados por parte de las empresas, los cuales pueden tener un impacto social o ambiental. La variable dependiente es la polaridad. $P_{0}$ es la polaridad de todos los tweets durante los días previos al evento y $P_{1}$ es la polaridad de todos los tweets en los días subsecuentes al evento. En este estudio utilizamos una ventana de tres días antes y después del evento. Los tweets son recolectados directamente del sitio Twitter Sentiment para su clasificación por fechas y empresa. Este sitio es resultado de un trabajo académico desarrollado por estudiantes de la Universidad de Stanford y clasifica en positivos y negativos los sentimientos expresados a través de tweets (Go et al., 2009). También permite obtener información histórica en un formato de hoja de cálculo de manera clara y concisa gracias a la explicación del funcionamiento del sitio. Debido a que se trata de un proyecto académico, el acceso es permitido de manera gratuita.

Como variable independiente se consideran los eventos de impacto social ocurridos durante el periodo del 25 de noviembre de 2009 al 24 de mayo de 2011. Se recopilaron eventos registrados en los reportes emitidos a nivel de empresa por Sustainalytics, organización internacional - con sede en Ámsterdam - que lleva a cabo investigaciones y análisis específicamente en temas de sostenibilidad y que se especializa en el análisis de factores ambientales, sociales y de gobernanza (ESG) en los ámbitos empresariales, institucionales o en el nivel país. Los reportes son utilizados como apoyo en el proceso de toma de decisiones para diversas instituciones financieras en todo el mundo (Gomez y Verma, 2012; Chelli y Gordin, 2012).

Además de reportar los incidentes a nivel de empresa, Sustainalytics permite identificar las fechas y fuentes de información de donde dichos incidentes provienen, lo que nos permite relacionar los tweets emitidos por los diferentes stakeholders con los incidentes emitidos en los reportes de esta compañía durante un mismo periodo de tiempo, los cuales consideran como fuentes de información los reportes realizados por parte de las mismas empresas, reportes en los medios de información, datos emitidos por organizaciones no-gubernamentales, estudios sectoriales, así como información proporcionada por instituciones públicas. 


\section{Recopilación de datos}

Debido a que no se cuenta con información histórica suficiente por parte de Twitter para poder realizar el presente análisis en empresas mexicanas, este primer estudio se enfoca en empresas norteamericanas. Se seleccionó diez empresas de manera aleatoria de acuerdo con los siguientes criterios: 1) son parte de las 50 empresas más admiradas en 2011 según Fortune (CNN Money, 2011); 2) Twitter Sentiment cuenta con datos por día durante el periodo del 25 de noviembre de 2009 al 24 de mayo de2011; 3) Sustainalytics cuenta con reportes a nivel empresa. Las compañías seleccionadas son Apple, Cisco, Coca Cola, Dell, Disney, Google, Nike, Pepsi, Starbucks y Walmart.

\section{Análisis de datos}

Se evaluaron los sentimientos de los tweets - que hacen referencia a incidentes causados directa o indirectamente por parte de las empresas y que tienen un efecto en la actitud de los stakeholders hacia ellas - como respuesta ante eventos de impacto social. Algunos ejemplos de eventos sociales pueden ser daño al medio ambiente atribuido a negligencia de la empresa; despido injustificado de empleados; actos discriminatorios relacionados con cuestiones raciales, sexuales, religiosas o de cualquier otra índole.

Para analizar las reacciones de los stakeholders ante estos eventos sociales se utilizaron algunos elementos de la metodología del estudio de eventos, desarrollada para medir el resultado de ciertas gestiones de la empresa y su efecto en el precio de las acciones de la misma (McWilliams et al., 1999). Los eventos principalmente estudiados en finanzas son los reportes de utilidades trimestrales, los nuevos productos o inversiones, los cambios regulatorios o legislativos, las inversiones o adquisiciones, y cualquier otro evento de significancia relacionado con las finanzas de la empresa.

Cabe destacar que esta metodología ha sido utilizada dentro del campo de la Responsabilidad Social Empresarial (CSR, por sus siglas en inglés). En algunos estudios se buscó medir el efecto de la des-inversión en Sudáfrica en el precio de las acciones de ciertas empresas (Meznar, Nigh y Kwok, 1994; Wright y Ferris, 1997), así como el impacto de eventos adversos sobre el valor de las acciones (Godfrey, Merrill y Hansen, 2008). Su uso ha sido criticado severamente por el hecho de que en la mayoría de los estudios realizados se considera la evaluación del impacto 
de los eventos hacia un sólo actor, los accionistas o inversionistas, dejando fuera todos los demás actores considerados como importantes dentro de los estudios de CSR (McWilliams y Siegel, 1997; McWilliams, Siegel y Teoh, 1999).

Sin embargo, más allá de las críticas que el estudio de eventos ha recibido referente a los estudios sobre CSR, esta metodología puede representar una forma apropiada de entender los sentimientos de los stakeholders ante incidentes realizados por parte de las empresas. Dada la naturaleza de nuestro estudio, se considera el impacto en los usuarios de Twitter que representan múltiples stakeholders, no únicamente el impacto sobre los accionistas de las empresas.

La metodología de estudio de eventos está basada en el modelo de índice del mercado. Utiliza los valores diarios de las acciones de las empresas, así como los valores del mercado, para determinar la tasa de rendimiento de las acciones de las empresas $i$ durante el periodo $t$ a nivel empresa. Cada uno de los eventos son estimados de la siguiente manera:

$$
\mathrm{R}_{i t}=\alpha_{i}+\beta_{i} \mathrm{R}_{m t}+\varepsilon_{i t}
$$

En donde $\mathrm{R}_{i t}$ es igual a la tasa de rendimiento de los precios de la empresa $i$ durante el periodo $t ; \mathrm{R}_{m t}$ representa la tasa de rendimiento del portafolio de las acciones del imercado en el periodo $t ; \alpha_{i}$ es el término de intersección; $\beta_{i}$ es el riesgo sistemático del mercado $i$; por último, $\varepsilon_{i t}$ es igual al error con $\mathrm{E}\left(\varepsilon_{i t}\right)=0$.

Para determinar la ecuación anterior es necesario calcular el rendimiento anormal (AR) diario de la empresa $i$, el cual es obtenido de la siguiente manera:

$$
A \mathrm{R}_{i t}=\mathrm{R}_{i t}-\left(\alpha_{i}+\mathrm{b}_{i} \mathrm{R}_{m t}\right)
$$

En donde $\alpha_{i} b_{i}$ representan los parámetros de los mínimos cuadrados ordinarios estimados de $\mathrm{R}_{i t}$ en $\mathrm{R}_{m t}$ durante el periodo precedente al evento. Los rendimientos anormales $\left(A \mathrm{R}_{i t}\right)$ representan la utilidad obtenida de la empresa después de haber sido ajustada por el proceso de ganancia normal en el periodo $t$. En otras palabras, esto representa que la tasa de utilidad de las acciones es ajustada después de haber sido extraída la utilidad esperada de la utilidad real. Cualquier cambio significativo puede ser considerado como un rendimiento anormal. 
La significancia en el promedio de los rendimientos anormales permite a los investigadores inferir si el evento estudiado tuvo un impacto significativo en el valor de la empresa. Sin embargo, para considerar lo anterior McWilliams y Siegel (1997) sugieren considerar los siguientes elementos: 1) los mercados son eficientes; 2) el evento que se estudia es analizado por primera vez; y 3) no existen otros eventos durante el periodo de análisis que puedan confundir los resultados del estudio. Este último punto es crucial para este trabajo, dado que si existe cualquier otro evento financiero relevante durante el periodo de estudio, puede dificultar que no se logre aislar el impacto sobre el precio de las acciones.

En el presente análisis no se consideran los “rendimientos anormales". En su lugar se estiman las "polaridades anormales" $(A P)$, que se obtuvieron a través del uso de una regresión local polinomial. Primero, se calcula la polaridad diaria de la siguiente manera:

$$
P=\frac{p x}{(p x+n x)} x 100
$$

En donde la polaridad $P$ es igual al número total de tweets positivos $(p x)$ dividido entre la suma de los tweets positivos ( $p x)$ y negativos $(n x)$; el resultado es multiplicado por cien para considerar los datos en términos de porcentaje. Entre más cercano sea a $100 \%$, mayor es la polaridad de sentimiento positivo; entre más cercano sea a $0 \%$, menor es la polaridad de sentimiento positivo, es decir, es mayor la polaridad de sentimiento negativo.

Las $A P$ se consideran como un cambio significativo en la polaridad de los sentimientos de los tweets detectados día a día con el uso de la regresión local polinomial. La regresión local polinomial es uno de los métodos no-paramétricos más utilizados en la investigación de datos no lineales, dadas sus propiedades asintóticas (Abberger, 2004). El objetivo de la regresión local polinomial es aproximar a un polinomio de grado $p$ alrededor de un punto determinado utilizando la información que rodea a dicho punto. En este caso, los cambios anormales en las polaridades de los sentimientos están dados por la siguiente ecuación:

$$
A P_{i t}=P_{i t}-\hat{P}_{i t}
$$

Donde $P_{i t}$ es la polaridad del sentimiento observado y $\widehat{P}_{i t}$ representa la regresión local polinomial estimada de $P_{i t}$ durante el periodo estimado precedente al evento. 
Para realizar lo anterior se utiliza el programa R para calcular la regresión local polinomial y la función loess (locally weighted scatterplot smoothing). Se calibró el modelo para estimar las 20 observaciones precursoras a cada punto $x_{i}$ tratando de predecir la probabilidad de $x_{21}$. Asimismo, dentro del modelo se considera como "anormal" todos aquellos puntos que fueran superiores a tres desviaciones estándar. Finalmente, para la prueba de hipótesis fue utilizada una tabla de contingencia de 2X2 con el propósito de determinar la existencia de cualquier relación entre las polaridades identificadas como anormales y los reportes de incidentes emitidos por Sustainalytics, puesto que los incidentes reportados en los medios pueden tener un impacto significativo en las polaridades de los tweets o:

H0: Los incidentes reportados en Sustainalytics son independientes de la polaridad de los tweets.

H1: Los incidentes reportados en Sustainalytics tienen relación con la polaridad de los tweets.

\section{Resultados}

Para cada una de las empresas se medió la polaridad de tweets durante 544 días; también se calculó la polaridad de los sentimientos considerando el número de tweets positivos y negativos emitidos por día. Para el total de las diez empresas fueron identificados 334 días con "incidentes o controversias" reconocidos y reportados por parte de Sustainalytics durante el periodo de investigación.

Por otra parte, utilizando el modelo de regresión local polinomial, fueron encontrados 337 observaciones o datos con polaridades de sentimientos de Twitter consideradas como "anormales"; de ellas $158(47 \%)$ reflejan un incremento en la polaridad del sentimiento y 179 (53\%) la reducen. Para ver un ejemplo de las polaridades anormales se puede observar el caso de Apple en la figura 1, donde las polaridades consideradas como anormales son marcadas con un punto gris sólido. 


\section{Figura 1}

\section{Identificación de polaridades anormales para el caso de Apple Inc}

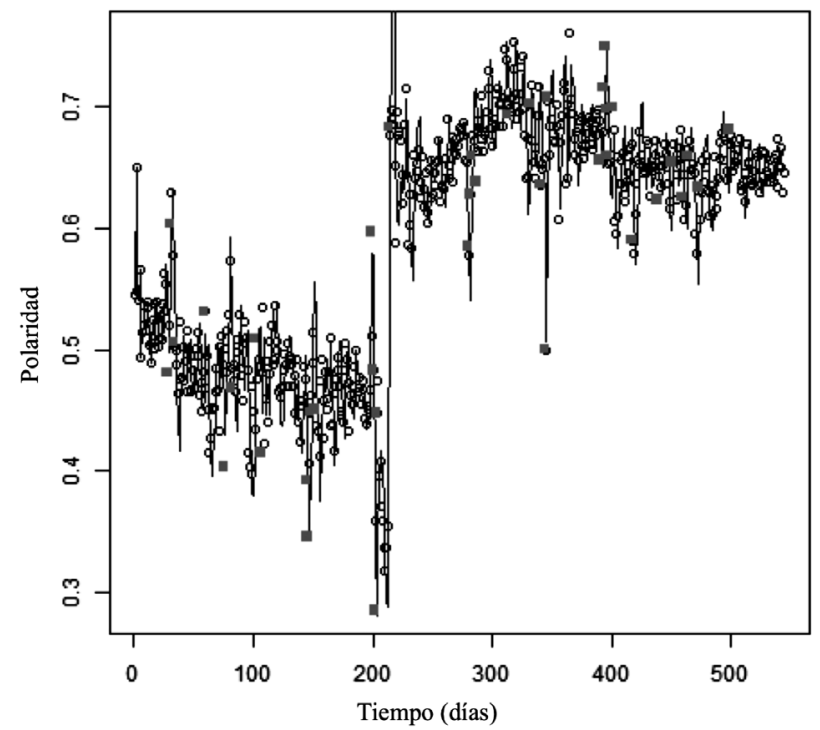

Fuente: Elaboración propia.

Cabe mencionar que durante el periodo del 5 al 6 de noviembre de 2010 se dio una re-programación del sitio "Twitter Sentiment”, lo que significó un cambio sustancial en las polaridades calculadas en toda la muestra del presente estudio; lo anterior se puede apreciar en la figura 1, pues a partir del día 200 se puede observar un dato "atípico", resultado de dicha configuración.

Durante el análisis se pudo observar que 45 datos identificados como anormales coinciden en fechas con los incidentes reportados por Sustainalytics. Al examinar la relación entre los datos anormales o las polaridades anormales y los eventos sociales, se encontró una relación altamente significativa, como se muestra en el cuadro 1 . 


\section{Cuadro 1}

Tabla de contingencia entre las polaridades anormales y los incidentes sociales

\begin{tabular}{c|c|c|c}
\hline & $\begin{array}{c}\text { Polaridad } \\
\text { anormal }\end{array}$ & $\begin{array}{c}\text { Polaridad } \\
\text { normal }\end{array}$ & Total \\
\hline Días con incidentes & 45 & 289 & 334 \\
\hline Días sin incidentes & 292 & 4,814 & 5,106 \\
\hline Total & 337 & 5,103 & 5,440 \\
\hline
\end{tabular}

Con una chi cuadrada de 32.44 y un valor de P de la prueba exacta de Fisher $<0.00$, se concluye que existe una significancia en la relación existente entre los incidentes reportados y las polaridades anormales de los tweets, lo cual apoya la hipótesis.

\section{Conclusiones}

Los resultados sugieren un escenario de "vaso medio lleno" respecto a la validez del uso de las polaridades anormales como un indicador de la reacción de los usuarios de Twitter ante los eventos de impacto social. Considerando las respuestas coincidentes en fechas de las polaridades anormales y su relación con los incidentes sociales o ambientales reportados, se encontró una gran significancia tanto estadística como cualitativa. Sin embargo, una gran cantidad de polaridades identificadas como aparentemente anormales $(86.6 \%)$ no corresponden a ningún incidente. Se considera que estos resultados son consistentes con la idea de que las polaridades anormales incluyen algunos elementos de "ruido" ante la presencia de incidentes sociales o ambientales.

Claramente existen limitaciones en la presente investigación. Primero, Sustainalytics no es perfecta, así como cualquier otra agencia calificadora; dichas agencias únicamente detectan y reportan los incidentes tal y como aparecen en los medios noticiosos y otras fuentes públicas de información. Twitter en la actualidad revela incidentes de impacto social que no son identificados por Sustainalytics, así que jamás habrá una asociación perfecta entre los eventos identificados por la agencia calificadora y las polaridades anormales. Por ejemplo, "sue rumor adob sdk rule -- http://bit.ly/bsIimd http://bit.ly/aJwke4 file corporatethi destin" es un tweet que hace mención a un posible acto legal en contra de Apple, publicado el día 15 de abril de 2010, y del cual no se tiene registro en los reportes de Sustainalytics. Este ejemplo lleva a la conclusión de que Twitter puede ser una herramienta para identificar eventos de impacto social que las agencias calificadoras sociales no detectarían, por lo menos no de manera inmediata. 
Segundo, la medición de la polaridad de los tweets presenta datos crudos, es decir, considera cualquier cantidad de comentarios relacionados con las empresas estudiadas, tales como "te veo frente a la puerta de Walmart", por lo que es necesario el desarrollo de filtros que permitan identificar aquellos tweets concernientes a los temas sociales y separarlos de aquellos considerados como solo "ruido".

A pesar de lo anterior, existe una gran área de oportunidad para continuar con esta línea de investigación. Este estudio demuestra de manera preliminar que tanto las redes sociales, en general, y Twitter, en particular, pueden proveer información útil para aquellos investigadores interesados en el estudio del desempeño social empresarial. Por el momento, con el enfoque de un "vaso medio lleno" se infiere que los datos agregados de las polaridades anormales pueden ser utilizados como una aproximación para la medición de la intensidad de los eventos de impacto social; por ejemplo, entre diferentes tipos de organización. En el futuro es necesario refinar el entendimiento de los mecanismos que generan los cambios en las polaridades y la relación de estos mismos mecanismos en el proceso que estimula a los stakeholders a realizar menciones en las redes sociales ante incidentes sociales o ambientales.

Este trabajo, asimismo, representa uno de los primeros esfuerzos que destacan el uso de las redes sociales como una herramienta que permite entender la importancia de uno de los muchos problemas de la administración: el desempeño social empresarial. Sin embargo, es sólo una de las muchas áreas que pueden ser estudiadas bajo este enfoque. Al considerar una forma innovadora de medir el CSP a través del análisis de los sentimientos en las redes sociales, este estudio aporta nuevos elementos para la ampliación de la literatura actual que hay acerca de su medición.

Lograr comprender el sentimiento de los stakeholders puede servir como apoyo para entender las diversas inquietudes relacionadas con el desempeño de una empresa; por ejemplo, la efectividad de sus estrategias en un contexto específico. Lo anterior puede generar una ventaja competitiva para las empresas que adoptan su uso porque permite limitar el uso de la información en aquella que es útil para los objetivos específicos de la empresa y no utilizar datos agregados para la generación de múltiples supuestos.

Esto representa un área de oportunidad para la aplicación de la inteligencia de negocio, ya que puede apoyar a las empresas en desarrollar sus estrategias de acuerdo a las necesidades especificas de cada una de sus unidades de negocio, mismas que 
pueden ir desde el desarrollo de sus estrategias de mercado, hasta el desarrollo de sus estrategias o iniciativas como CSR a nivel local.

Por otra parte, el presente estudio también puede aportar elementos de valor en trabajos acerca de la justicia organizacional. Bajo la premisa de que las empresas dependen de una eficiente interacción y validación por parte de sus stakeholders, entender la perspectiva de terceros, su percepción de justicia ante eventos sociales y cómo éstos se manifiestan a través de las redes sociales, tiene varias implicaciones. Por un lado, puede generar valor en la realización de las estrategias empresariales ante eventos específicos; por otro, también representa una fuente importante de investigación que permite ampliar la literatura actual sobre justicia organizacional.

De la misma manera, este estudio puede ampliar la recurrente búsqueda que pretende relacionar el CSP y el CFP (Alonso-Almeida et al., 2012). Debido a que gran parte de la investigación se ha enfocado en buscar la relación entre el precio de las acciones y el cambio en las polaridades de los tweets, el próximo paso consiste en determinar la relación entre los sentimientos de los stakeholders y su relación con los sentimientos de los accionistas.

Existe un creciente interés en el estudio de las redes sociales y su relación con múltiples actividades cotidianas, por lo que no se puede dejar de lado su relevancia para el estudio del CSP. Actualmente es posible tener acceso a múltiples fuentes de información que pueden destacar la relevancia o desempeño de las empresas ante contextos específicos; eso representa la percepción de millones de stakeholders que se comportan de manera dinámica ante eventos que pueden estar directa e indirectamente relacionados con la actividad de las empresas.

\section{Referencias}

Abberger, K. (2004). Nonparametric regression and the detection of turning points in the Ifo Business Climate. CESifo Working Paper 1283.

Adams, J. S. (1965). Inequity in social exchange. En L. Berkowitz (ed.). Advances in experimental social psychology 2. New york: Academic Press: 267-299. 
Alonso-Almeida, M. M., M. P. Rodríguez García, K. A. Cortez Alejandro y J. L. Abreu Quintero (2012). La responsabilidad social corporativa y el desempeño financiero: un análisis en empresas mexicanas que cotizan en la bolsa. Contaduría y Administración 57 (1): 53-77.

Bennett, Sh. (2012). Twitter now seeing 400 million tweets per day, increased mobile ad revenue, says CEO. En All Twitter, the unofficial Twitter resource. Disponible en: http://www.mediabistro.com/alltwitter/twitter-400-milliontweets_b23744

Berman, S. L., A. C. Wicks, S. Kotha y T. M. Jones (1999). Does stakeholder orientation matter? The relationship between stakeholder management models and firm financial performance. Academy of Management Journal 42: 488506.

Blau, P. (1964). Exchange and power in social life. New York: Wiley.

Bosse, D., R. Phillips y J. Harrison (2009). Research notes and commentaries: stakeholders, reciprocity and firm performance. Strategic Management Journal 30: 447-456.

Briseño García, A., J. Lavín Verástegui y F. García Fernández (2011). Análisis exploratorio de la responsabilidad social empresarial y su dicotomía en las actividades sociales y ambientales de la empresa. Contaduría y Administración 233: 73-90.

Clarkson, M. (1995). A stakeholder framework for analyzing and evaluating corporate social performance. Academy of Management Review 20 (1): 92-117.

(1988). Corporate social performance in Canada, 1976-86. En L. E. Preston (comp.). Research in Corporate Social Performance and Policy 19. Greenwich: JAI Press: 241-265.

Chelli, M e Y. Gendron (2012). Sustainability ratings and the disciplinary power of the idelolgy of numbers. Journal of Business Ethics. DOI 10.1007/s10551012-1252-3. 
CNN (2011). World's most admired companies, Top 50. Disponible en: http://money.cnn.com/magazines/fortune/mostadmired/2011/full_list/

Dugan, L. (2012). Twitter surpasses 500 million registered users on wednesday. En All Twitter, The unofficial Twitter resource. Disponible en: http://www. mediabistro.com/alltwitter/500-million-registered-users_b18842

Folger, R. (2001). Fairness as deonance. En S. W. Gilliand, D. D. Steiner y D. P. Skarlicki (eds.). Research in Sociallssues in Management. Charlotte, NC: Information Age: 3-31.

Fink, C., D. Chou, J. Kopecjy y A. Llorens (2011). Coarse- and fine- grained sentiment analysis of social media text. Johns Hopkins APL Technical Digest (30) 1: 22-30.

Freeman, R. E. (1984). Strategic Management: A stakeholder approach. Boston: Pitman.

Go, A., L. Huang y R. Bhayani (2009). Sentiment analysis of Twitter data. Disponible en: http://nlp.stanford.edu/courses/cs224n/2009/fp/3.pdf

Godfrey, P. C., C. B. Merrill y J. M. Hansen (2008). The relationship between corporate social responsibility and shareholder value: an empirical test of the risk management hypothesis. Strategic Management Journal 30: 425-445

Gómez, R. y A. Verma (2012). Corporate social responsibility and greater worker protection: two sides of the same coin? Center for Industrial Relations and Human Resources and Woodsworth College, Toronto, ON, Cadada.

Harrison, J. S. y R. E. Freeman (1999). Stakeholders, social responsibility, and performance: empirical evidence and theoretical perspectives. Academy of Management Journal 42: 479-485.

D. A. Bosse y R. A. Phillips (2010). Managing for stakeholders, stakeholder utility functions, and competitive advantage. Strategic Management Journal 31: 58-74. 
Husted, B. (2000). A contingency theory of corporate social performance. Business and Society 39 (1): 24-48.

Jensen, B. J., M. Zhang, K. Sobel y A. Chowdury (2009). Twitter power: tweets as electronic word of mouth. Journal of the American Society for Information Science and Technology 60 (11): 2169-2188.

Kale, A., A. Karandikar, P. Kolari, A. Java, T. Finin y A. Joshi (2007). Modeling trust and influence in the blogosphere using link polarity, en The proceedings of International Conference on Weblogs and Social Media, ICWSM.

Kaplan, A. M. y M. Haenlein (2010). Users of the world, unite! The challenges and opportunities of social media. Business Horizons 53 (1): 59-68.

Leventhal, G. (1976). Fairness in social relationships. En J. Thibaut, J. Spence y R. Carson (eds.). Contemporary topics in social psychology. Morristown: General Learning Press, 211-249.

Lind, E. A. y T. R. Tyler (1988). The social psychology of procedural justice. New York: Plenum Perss.

Luoma, P. y J. Goodstein (1999). Stakeholders and corporate boards: institutional influences on board composition and structure. Academy of Management Journal 42 (5): 553-563.

McWilliams, A. y D. Siegel (1997). Event studies in management research: theoretical and empirical issues. Academy of Management Journal 40 (3): 568-592.

, D. Siegel y S. H. Teoh (1999). Issues in the use of the event study methodology: a critical analysis of corporate social responsibility studies. Organizational Research Methods 2 (4): 340-365.

Merlo, R., D. Contreras y C. Puente (2010). Análisis de opiniones en Internet a partir de la red social Twitter. Anales de mecánica y electricidad noviembre-diciembre: 54-59. 
Meznar, M., D. Nigh y C. Kwok (1994). Effect of announcements of withdrawal from South Africa on stockholder wealth. Academy of Management Journal 37 (6): 1636-1648.

Mitnick, B. M. (2000). Commitment, revelation, and the testaments of belief: the metrics of measurement of corporate social performance. Business and Society 39 (4): 419-465.

O’Connor, B., R. Balasubramanyan, B. Routlrfgr y N. Smith, N. (2010). From tweets to pPlls: Linking text sentiment to public opinion time series, Proceedings of the International Association for the Advancement of Artificial Intelligence Conference on Washington, DC, May 2010.

Organization for Economic Cooperation and Development. (2007). Participative Web: User-Created content. Disponible en: http://www.oecd.org/ newsearch/0,3766,en_2649_201185_1_1_1_1_1,00.html?q=UGC\&sa=Search\&cx $=012432601748511391518 \% 3$ Axzeadub0b0a\&cof=FORID\%3A11\&ie $=$ UTF-8

O'Reilly, J. y K. Aquino (2011). A model of third parties' morally motivated responses to mistreatment in organizations. Academy of Management Review 36 (3): 526-543.

Orlitzky, M. y D. L. Swanson (2012). Assessing stakeholder satisfaction: toward a supplemental measure of corporate social performance as reputation. Corporate Reputation Review 15 (2): 119-137.

Pang, B. y L. Lee (2008). Opinion, mining and sentiment analysis. Foundations and Trends in Information Retrieval 2: 1-135.

Preston, L. E. (1990). Stakeholder management and corporate performance. Journal of Behavioral Economics 19 (4): 361-375.

Rowley, T. y S. Berman (2000). A brand new brand of corporate social performance. Business and Society 39 (4): 397-418. 
Rupp, D., J. Ganapathi, R. Aguilera y C. Williams (2006). Employee reactions to corporate social responsibility: an organizational justice framework. Journal of Organizational Behavior 27:537-543.

Sheppard, B. H., R. J. Lewicki y J. W. Minton (1992). Organizational justice: the search for fairness in the workplace. New York: Lexington Books.

Skarlicki, D. P. y C. T. Kulik (2005). Third-party reaction employee (mis)treatment: a justice perspective. Research in Organizational Behavior 26: 183-229.

Thelwall, M. y R. Prabowo (2007). Identifying and characterizing public science-related fears from RSS feeds. Journal of the American Society for Information Science and Technology 58 (3): 379-390.

K. Buckley y G. Paltoglou (2011). Sentiment in Twitter events. Journal of the American Society for Information Science and Technology 62 (2): 406-418.

Thibaut, J. y L. Walker (1975). Procedural justice: a psychological analysis. Hillsdale, NJ: Erlbaum.

Thongsuk, C., C. Haruechaiyasak y S. Somkid (2011). Classifying business types on Twitter based user influential analysis, en The proceedings of the International Conference on Future Informational Technology, IPCSIT.

Turillo, C., R. Folger, J. Lavelle, E. Umphress y J. Gee (2002). Is virtue its own reward? Self-sacrificial decisions for the sake of fairness. Organizational Behavior and Human Decision Processes 89: 839-865.

Waddock, S. y S. Graves (1997). The corporate social performance-financial performance link. Strategic Management Journal 18 (4): 303-319.

Wartick, S. y J. Mahon (2009). Corporate social performance profiling: the importance of multiple stakeholder perspectives. Paper presented at The Annual Meeting of the International Association for Business and Society, Snowmass, CO, June 18-21. 
Wood, D. y R. Jones (1995). Stakeholder mismatching: a theoretical problem in empirical research on corporate social performance. International Journal of Organizational Analysis 3: 229-267.

Wright, P. y S. Ferris (1997). Agency conflict and corporate strategy: the effect of divestment on corporate value. Strategic Management Journal 18 (1): 77-83. 
